JOURNAL OF THE

AMERICAN MATHEMATICAL SOCIETY

Volume 16, Number 1, Pages 123-138

S 0894-0347(02)00406-X

Article electronically published on October 9, 2002

\title{
THE NATURE OF SINGULARITIES IN MEAN CURVATURE FLOW OF MEAN-CONVEX SETS
}

\author{
BRIAN WHITE
}

\section{INTRODUCTION}

Let $K$ be a compact subset of $\mathbf{R}^{n+1}$, or, more generally, of an $(n+1)$-dimensional riemannian manifold. We suppose that $K$ is mean-convex. If the boundary of $K$ is smooth and connected, this means that the mean curvature of $\partial K$ is everywhere nonnegative (with respect to the inward unit normal) and is not identically 0 . More generally, it means that $F_{t}(K)$ is contained in the interior of $K$ for $t>0$, where $F_{t}(K)$ is the set obtained by letting $K$ evolve for time $t$ under the level set mean curvature flow. As $K$ evolves, it traces out a closed set $\mathcal{K}$ of spacetime:

$$
\mathcal{K}=\left\{(x, t) \in \mathbf{R}^{n+1} \times \mathbf{R}: x \in F_{t}(K)\right\} .
$$

Also, there is associated to $\mathcal{K}$ a Brakke flow

$$
\mathcal{M}: t \mapsto M_{t}
$$

of rectifiable varifolds. We call the pair $(\mathcal{M}, \mathcal{K})$ a mean-convex flow.

Let $X=(x, t)$ be a point in spacetime with $t>0$. Suppose $\left(x_{i}, t_{i}\right)$ is a sequence of points converging to $X$ and $\lambda_{i}$ is a sequence of positive numbers tending to infinity. Translate the pair $\mathcal{M}$ and $\mathcal{K}$ in spacetime by

$$
(y, \tau) \mapsto\left(y-x_{i}, \tau-t_{i}\right)
$$

and then dilate parabolically by

$$
(y, \tau) \mapsto\left(\lambda_{i} y, \lambda_{i}^{2} \tau\right)
$$

to get new flows $\mathcal{M}_{i}$ and $\mathcal{K}_{i}$. The sequence $\left(\mathcal{M}_{i}, \mathcal{K}_{i}\right)$ is called a blow-up sequence at $X$. General compactness theorems guarantee that this sequence will have subsequential limits. A subsequential limit $\left(\mathcal{M}^{\prime}, \mathcal{K}^{\prime}\right)$ is called a limit flow. Here

$$
\mathcal{M}^{\prime}: t \in(-\infty, \infty) \mapsto M_{t}^{\prime}
$$

is a Brakke flow and

$$
\mathcal{K}^{\prime}: t \in(-\infty, \infty) \mapsto K_{t}^{\prime}
$$

is a "weak set flow" W4 $§ 2.2$ ], W2] (or "set-theoretic subsolution" in the terminology of [1] and [12]).

Received by the editors November 25, 1998 and, in revised form, September 11, 2002.

2000 Mathematics Subject Classification. Primary 53C44; Secondary 49Q20.

Key words and phrases. Mean curvature flow, mean convex, singularity.

The research presented here was partially funded by NSF grants DMS 9803403, DMS 0104049, and by a Guggenheim Foundation Fellowship. 
Let $T^{*}$ be the first time that singularities occur in the flow $(\mathcal{M}, \mathcal{K})$. For analyzing the singularities that occur at time $T^{*}$, it is useful to consider the special case when each $t_{i}$ is $\leq T^{*}$. A limit flow obtained in this way is called a special limit flow.

If $\left(x_{i}, t_{i}\right) \equiv(x, t)$, then the limit flow $\left(\mathcal{M}^{\prime}, \mathcal{K}^{\prime}\right)$ is called a tangent flow to $(\mathcal{M}, \mathcal{K})$ at $X$. Every tangent flow is "backwardly self-similar" in that

$$
K_{-r^{2}}^{\prime} \equiv(r)_{\#} K_{-1}^{\prime}
$$

and

$$
M_{-r^{2}}^{\prime} \equiv(r)_{\#} M_{-1}^{\prime}
$$

for $r \geq 0$. (See [W3, §7] or [13].) Here $(r)_{\#}: z \mapsto r z$ denotes dilation by $r$.

In this paper we prove

Theorem 1. Let $(\mathcal{M}, \mathcal{K})$ be a mean-convex flow in $\mathbf{R}^{n+1}$ or in an $(n+1)$-dimensional riemannian manifold. Let $\left(\mathcal{M}^{\prime}, \mathcal{K}^{\prime}\right)$ be any limit flow if $n<7$, or a special limit flow if $n \geq 7$. Then $K_{t}^{\prime}$ is convex for every $t$, and there is a $T \leq \infty$ such that

(1) $K_{t}^{\prime}$ has interior points if and only if $t<T$.

(2) $\partial K_{t}^{\prime}$ is smooth for $t<T$.

(3) $K_{t}^{\prime}=\emptyset$ for $t>T$.

Furthermore, if $\left(\mathcal{M}^{\prime}, \mathcal{K}^{\prime}\right)$ is backwardly self-similar, then it is either (i) a static multiplicity 1 plane or (ii) a shrinking sphere or cylinder.

(We say that $\left(\mathcal{M}^{\prime}, \mathcal{K}^{\prime}\right)$ is a shrinking sphere (cylinder) if $\partial K_{t}^{\prime}$ is a sphere (cylinder) for all $t<0$.)

Corollary. Let $\left(x_{i}, t_{i}\right)$ be a sequence of regular points of $(\mathcal{M}, \mathcal{K})$ converging to a singular point $(x, t)$. If $n \geq 7$, assume that $t_{i} \leq T^{*}$, where $T^{*}$ is the first time that singularities occur. Let $h_{i}$ be the scalar mean curvature of $M_{t_{i}}$ at $x_{i}$. Translate $M_{t_{i}}$ by $-x_{i}$ and then dilate by $h_{i}$ to get a surface $\tilde{M}(i)$. Then a subsequence of the $\tilde{M}(i)$ converges smoothly on compact sets to a smooth convex surface $\tilde{M}$.

The proofs of Theorem 1 and its corollary are completed in the penultimate section ("Conclusion") of this paper.

Theorem 1 does not completely characterize the class of limit flows to meanconvex flows. For example the grim reaper flow in $\mathbf{R}^{2}$ (or its cartesian product with $\mathbf{R}^{n-1}$ in $\mathbf{R}^{n+1}$ ) satisfies all the conclusions of Theorem 1, but does not arise as a limit flow (or special limit flow if $n \geq 7$ ) to any mean-convex flow (see Corollary 4 to Theorem 5). (The higher-dimensional analogs of the grim reaper that have more rotational symmetry do occur as limit flows to mean-convex flows.) Some conjectures that go beyond Theorem 1 are listed at the end of the paper.

Gerhard Huisken and Carlo Sinestrari [HS1], HS2] recently proved a result very similar to the corollary above by a different method. However, even in low dimensions their proof requires that $t_{i}<T^{*}$. Also, their result requires that the sequence $\left(x_{i}, t_{i}\right)$ be chosen in a particular way.

\section{PRELIMINARIES}

This paper relies very heavily on [W4]. For basic properties of mean-convex flows, limit flows, and tangent flows, the reader is referred to the first few sections of that paper. We will also need the following two results, which are local consequences of the strict maximum principle. 
Theorem 2 (strict maximum principle for $h$ ). Let $\mathcal{M}$ be a Brakke flow of hypersurfaces in $\mathbf{R}^{n+1}$. Suppose the mean curvature is $\geq 0$ at every regular point, and is 0 at some regular point $X=(x, \tau)$. Then there is a neighborhood $W$ of $x$, a smooth connected minimal hypersurface $S$ properly embedded in $W$, and an $\epsilon>0$ such that

$$
M_{t} \cap W=S
$$

for $\tau-\epsilon \leq t \leq \tau$.

Proof. Since $X$ is a regular point, we can parametrize $\mathcal{M}$ near $X$ by a mapping

$$
(u, t) \in U \times(\tau-\delta, \tau+\delta) \rightarrow(F(u, t), t)
$$

where $U$ is an open set in $\mathbf{R}^{n}$ and

$$
\frac{\partial}{\partial t} F=\Delta F
$$

(Here $\Delta$ denotes the laplacian with respect to the metric induced by $F$; that is, the pull-back by $F$ of the euclidean metric on $\mathbf{R}^{n+1}$.) Huisken [H1] shows that the scalar mean curvature $h(x, t)$ satisfies

$$
\frac{\partial}{\partial t} h=\Delta h+|A|^{2} h
$$

where $A$ is the second fundamental form at $F(u, t)$.

Then by the strict maximum principle, $h \equiv 0$ on $U \times(\tau-\delta, \tau]$. Thus $\frac{\partial}{\partial t} F \equiv 0$ on $(\tau-\epsilon, \tau]$ so $F$ is independent of $t$ in this set:

$$
F(u, t) \equiv \phi(u) .
$$

But this immediately implies the desired result.

Remark (not needed in this paper). The reader may wonder why the conclusion of Theorem 1 is only asserted for $t \leq \tau$. The reason is that the theorem and its proof are essentially local, and to conclude that $M_{t} \cap W=S$ for $t>\tau$ would require a very different global argument. Indeed, that conclusion would be false in some settings. Consider for example motion by mean curvature of a manifold with boundary, the motion of the boundary being prescribed. If the boundary is still for $t \leq \tau$ and then starts moving, the conclusion of Theorem 2 will typically not hold for $t>\tau$.

For the next result, we need some notation. If $M$ is an $n$-dimensional hypersurface and $x$ is a regular point of $M$, we let

$$
G(M, x)=\frac{\kappa_{1}(x)}{h(x)}
$$

provided $h(x)>0$, where

$$
\kappa_{1}(x) \leq \kappa_{2}(x) \leq \ldots \leq \kappa_{n}(x)
$$

are the principal curvatures of $M$ at $x$ and

$$
h(x)=\sum_{i=1}^{n} \kappa_{i}(x)
$$

is the mean curvature.

If $h(x)=0$, then $\kappa_{1}(x) \leq 0$; in this case, we let $G(M, x)$ be $-\infty$ or undefined according to whether $\kappa_{1}(x)$ is negative or 0 . Note that if a sequence of surfaces 
$M_{i}$ converges smoothly to $M$ and $x_{i} \in M_{i}$ converges to $x \in M$, then $G\left(M_{i}, x_{i}\right)$ converges to $G(M, x)$ unless $G(M, x)$ is undefined.

If $X=(x, t)$ is a regular point of a flow $\mathcal{M}$ of hypersurfaces, then we let

$$
G(\mathcal{M}, X)=G\left(M_{t}, x\right) .
$$

Theorem 3 (strict maximum principle for $G$ ). Let $\mathcal{M}$ be a mean curvature flow of hypersurfaces in $\mathbf{R}^{n+1}$. Suppose there is an open subset $U$ of spacetime such that $\mathcal{M}$ is smooth in $U$ and such that the mean curvature is strictly positive in $U$. Suppose also that there is an $X_{0}=\left(x_{0}, t_{0}\right)$ in $U$ such that

$$
G\left(\mathcal{M}, X_{0}\right)=\min \left\{G(\mathcal{M}, X): X=(x, t) \in U \text { and } t \leq t_{0}\right\} .
$$

Then $G$ is a nonnegative constant for $t \leq t_{0}$ in a spacetime neighborhood of $X_{0}$.

Proof. Let $\gamma=G\left(\mathcal{M}, X_{0}\right)$. The result follows from Hamilton's strict maximum principle [Ha, §8] for tensors, applied to the tensor

$$
T(u, v)=A(u, v)-\gamma h u \cdot v
$$

where $h$ is the mean curvature, $\gamma=G\left(\mathcal{M}, X_{0}\right)$, and

$$
A: \operatorname{Tan} M \times \operatorname{Tan} M \rightarrow \mathbf{R}
$$

is the second fundamental form.

Readers who are not quite conversant both with Hamilton's paper and with the equations in Huisken's paper [H1] may find it troublesome to verify that the tensor $T$ in fact satisfies the hypotheses of Hamilton's theorem. For the benefit of those readers, a more self-contained proof (along the same lines) of Theorem 3 is given in the appendix to this paper.

\section{FIRST RESULTS}

Throughout this paper, we will let $(\mathcal{M}, \mathcal{K})$ be a mean-convex flow in an $(n+1)$ manifold. Fix some spacetime point $Z$ with positive time coordinate and let

$$
\mathcal{F}= \begin{cases}\{\text { all limit flows at } Z\} & \text { if } n<7, \\ \{\text { all special limit flows at } Z\} & \text { if } n \geq 7 .\end{cases}
$$

Note that the class $\mathcal{F}$ is compact and closed under parabolic dilations. If $n<7, \mathcal{F}$ is also closed under all spacetime translations:

$$
(x, t) \in \mathbf{R}^{n+1} \times \mathbf{R} \mapsto(x+u, t+v) .
$$

If $n \geq 7$, it is closed under those spacetime translations $\left(^{*}\right)$ with $v \geq 0$.

Theorem 4. The class $\mathcal{F}$ does not contain any static singular cones.

Proof for $n<7$. Any such cone corresponds to a stable one-sided area minimizing hypercone in $\mathbf{R}^{n+1}$. But there are no such cones if $n<7$. (See [W4, §8.7].)

Proof for $n \geq 7$. For the case $n \geq 7$, we observe that by the strong maximum principle for $h$ (Theorem 2), the mean curvature of $M_{t}$ is strictly positive for $0<$ $t<T^{*}$. This implies (by compactness of $M_{t}$ ) that the quantity

$$
G(t):=\inf \left\{G\left(M_{t}, x\right): x \in M_{t}\right\}
$$


is $>-\infty$ for each $t \in\left(0, T^{*}\right)$. The maximum principle for $G$ (Theorem 3 ) then implies that $G(t)$ is increasing on $\left(0, T^{*}\right)$. In particular, there is a $\Gamma \in \mathbf{R}$ such that

$$
G\left(M_{t}, x\right) \geq \Gamma
$$

for all $x \in M_{t}$ and $t<T^{*}$.

Now suppose, contrary to the theorem, that $\mathcal{F}$ contains a nonplanar static minimal cone $\left(\mathcal{M}^{\prime}, \mathcal{K}^{\prime}\right)$, so that

$$
M_{t}^{\prime} \equiv M^{\prime}
$$

where $M^{\prime}$ is a minimal cone. Let $\left(\mathcal{M}_{i}, \mathcal{K}_{i}\right)$ be a special blow-up sequence converging to $\left(\mathcal{M}^{\prime}, \mathcal{K}^{\prime}\right)$. The mean-convexity implies that $M^{\prime}$ is stable and one-sided area minimizing, which implies that its singular set has dimension $\leq n-7$ [W4, §8.7]. This in turn implies that there is a regular point $x$ of $M^{\prime}$ at which the second fundamental form is nonzero. Thus $h(x)=0$ and $\kappa_{1}(x)<0$, so

$$
G\left(M^{\prime}, x\right)=-\infty
$$

where $G$ is as in Theorem 3 .

Now $\mathcal{M}^{\prime}$ is smooth in a spacetime neigborhood $U$ of $X=(x, 0)$. Then the $\mathcal{M}_{i}$ converge smoothly to $\mathcal{M}$ in a neighborhood of $X$. (This is by the Brakke regularity theorem ([B] or $[\mathrm{W} 5])$ if the multiplicity of $\mathcal{M}^{\prime}$ near $X$ is 1 , and is by the "sheeting theorem" [W4, §8.1] if the multiplicity is 2 . In the latter case, the convergence is two-sheeted. No other multiplicities are possible [W4, §3.9].)

Now let $X_{i}$ be a sequence of points in the support of $\mathcal{M}_{i}$ that converges to $X$. By the smooth convergence, $X_{i}$ will be a regular point of $\mathcal{M}_{i}$ for all sufficiently large $i$, and

$$
G\left(\mathcal{M}_{i}, X_{i}\right) \rightarrow G\left(\mathcal{M}^{\prime}, X\right) .
$$

But $G\left(\mathcal{M}_{i}, X_{i}\right) \geq \Gamma>-\infty$ by (11), whereas $G\left(\mathcal{M}^{\prime}, X\right)=-\infty$ by (2). The contradiction proves the theorem.

Theorem 5. Let $\left(\mathcal{M}^{\prime}, \mathcal{K}^{\prime}\right)$ be in $\mathcal{F}$. Suppose a tangent flow to $\left(\mathcal{M}^{\prime}, \mathcal{K}^{\prime}\right)$ at some point $X$ is a static or quasistatic plane. Then the tangent flow must be a static multiplicity 1 plane, and hence $X$ must be a regular point of $\left(\mathcal{M}^{\prime}, \mathcal{K}^{\prime}\right)$.

Proof. If there were a spacetime point of multiplicity $>1$, there would be such a point $(x, t)$ with $t<0$ [W4, $\S 12.2]$. But then by W4, $\S 12.3]$, the class $\mathcal{F}$ would contain a static singular minimal cone. (Theorem 12.3 of W4 is stated only for limit flows, but the proof also works for special limit flows.) But by Theorem $4, \mathcal{F}$ does not contain any such cones.

Thus the tangent flow must be a static or quasistatic multiplicity 1 plane. But quasistatic multiplicity 1 planes cannot occur as limit flows of mean-convex flows W4 §5.4]. Hence the tangent flow is a static multiplicity 1 plane. Thus by Brakke's regularity theorem, $X$ is a regular point of $\left(\mathcal{M}^{\prime}, \mathcal{K}^{\prime}\right)$.

Corollary 1. Let $\left(\mathcal{M}^{\prime}, \mathcal{K}^{\prime}\right)$ be a flow in $\mathcal{F}$. Then there is a $T \leq \infty$ such that $K_{t}^{\prime}$ has nonempty interior for $t<T$, and $K_{t}^{\prime}=\emptyset$ for $t>T$.

Proof. Suppose $M_{t}^{\prime} \neq 0$. Then there is a point $(x, t)$ at which one of the tangent flows is a static or quasistatic plane. By the theorem, it must be a multiplicity 1 plane, which means (by Brakke regularity) that $(x, t)$ is a regular point. Thus $M_{t}$ is (near $x$ ) a smooth hypersurface with the interior of $K_{t}^{\prime}$ on one side and the complement of $K_{t}^{\prime}$ on the other. Thus $K_{t}^{\prime}$ has interior points. 
Corollary 2. Suppose $\left(\mathcal{M}^{\prime}, \mathcal{K}^{\prime}\right)$ is a backwardly self-similar flow in $\mathcal{F}$ and $X=$ $(x, t)$ is a point in the support of $\mathcal{M}^{\prime}$ with $t<0$. Then $X$ is a regular multiplicity 1 point of $\left(\mathcal{M}^{\prime}, \mathcal{K}^{\prime}\right)$. Thus the $t<0$ portion of $\left(\mathcal{M}^{\prime}, \mathcal{K}^{\prime}\right)$ is everywhere regular.

Proof. Let $\left(\mathcal{M}^{\prime \prime}, \mathcal{K}^{\prime \prime}\right)$ be a tangent flow to $\left(\mathcal{M}^{\prime}, \mathcal{K}^{\prime}\right)$ at $X$. Then $\left(\mathcal{M}^{\prime \prime}, \mathcal{K}^{\prime \prime}\right)$ is also in $\mathcal{F}$. By the self-similarity of $\left(\mathcal{M}^{\prime}, \mathcal{K}^{\prime}\right)$, this tangent flow must be a static minimal cone. Thus by Theorem 4 , it is a static plane. Hence by Theorem $5, X$ is a regular point of $\left(\mathcal{M}^{\prime}, \mathcal{K}^{\prime}\right)$.

Corollary 3. Suppose $\left(\mathcal{M}^{\prime}, \mathcal{K}^{\prime}\right)$ is in $\mathcal{F}$. Then the singular set of $\left(\mathcal{M}^{\prime}, \mathcal{K}^{\prime}\right)$ has parabolic Hausdorff dimension $\leq n-1$, and for almost every $t,\left(\operatorname{sing} \mathcal{M}^{\prime}\right)_{t}$ has dimension $\leq n-3$.

Proof. No static or quasistatic singular polyhedral cones occur as tangent flows to $\left(\mathcal{M}^{\prime}, \mathcal{K}^{\prime}\right)$ by [W4, $\left.\S 8.7\right]$. And by Theorem 5 , no static or quasistatic planes occur as tangent flows at any singular point. Then the general stratification (or dimension reducing) theory [W3, §9] gives the asserted bounds on dimension.

Corollary 4. Let $\left(\mathcal{M}^{\prime}, \mathcal{K}^{\prime}\right)$ be the "grim reaper" flow:

$$
\mathcal{K}^{\prime}=\left\{(x, t): x_{2} \leq \ln \left(\cos x_{1}\right)-t,-1<x_{1}<1\right\} .
$$

Then $\left(\mathcal{M}^{\prime}, \mathcal{K}^{\prime}\right)$ is not in $\mathcal{F}$.

Proof. Let $\left(\mathcal{M}^{\prime \prime}, \mathcal{K}^{\prime \prime}\right)$ be the tangent flow at infinity to $\left(\mathcal{M}^{\prime}, \mathcal{K}^{\prime}\right)$. (In other words, dilate $\left(\mathcal{M}^{\prime}, \mathcal{K}^{\prime}\right)$ parabolically by $1 / r$ and let $r \rightarrow \infty$ : the resulting limit is $\left(\mathcal{M}^{\prime \prime}, \mathcal{K}^{\prime \prime}\right)$.) Then $\left(\mathcal{M}^{\prime \prime}, \mathcal{K}^{\prime \prime}\right)$ is a quasistatic multiplicity two plane. If $\left(\mathcal{M}^{\prime}, \mathcal{K}^{\prime}\right)$ were in $\mathcal{F}$, then $\left(\mathcal{M}^{\prime \prime}, \mathcal{K}^{\prime \prime}\right)$ would be also. But by Theorem $5,\left(\mathcal{M}^{\prime \prime}, \mathcal{K}^{\prime \prime}\right)$ is not in $\mathcal{F}$.

Theorem 6. Let $\left(\mathcal{M}^{\prime}, \mathcal{K}^{\prime}\right)$ be a flow in $\mathcal{F}$ such that the mean curvature of $M_{t}^{\prime}$ vanishes at $x$ for some regular point $(x, t)$. Then $\left(\mathcal{M}^{\prime}, \mathcal{K}^{\prime}\right)$ is a a static multiplicity 1 plane. That is, there is a half-space $H$ of $\mathbf{R}^{n+1}$ such that

$$
\mathcal{K}^{\prime}=H \times \mathbf{R}
$$

and such that for every $t, M_{t}^{\prime}$ is the multiplicity 1 varifold associated with $\partial H$.

Proof. By the strict maximum principle for $h$ (Theorem 2), $(x, \tau)$ is a regular point with mean curvature 0 for $\tau$ in some interval $(t-\epsilon, t]$. By Corollary 3 to Theorem 5 , we can choose $\tau$ so that

$$
\left(\operatorname{sing} \mathcal{M}^{\prime}\right)_{\tau}
$$

has dimension $\leq n-3$.

By Theorem 1, there is an entire connected component $C$ of the regular set of $M_{\tau}^{\prime}$ on which the mean curvature vanishes.

Let $\operatorname{sing} \mathcal{M}^{\prime}$ be the set of spacetime singularities of $\mathcal{M}^{\prime}$. Let $Y$ be a point in $C \times(-\infty, \tau]$. Since $\operatorname{sing} \mathcal{M}^{\prime}$ has parabolic dimension $\leq n-1\left(\right.$ and $\left(\operatorname{sing} \mathcal{M}^{\prime}\right)_{t}$ has dimension $\leq n-3$ ) and since $C$ is pathwise connected, it follows that there is a spacetime curve:

$$
\gamma:[0,1] \rightarrow \mathbf{R}^{n+1} \times \mathbf{R}
$$

such that $\gamma(0)=Y, \gamma(1)=(x, t)$, and such that the time component of $\gamma(\cdot)$ is monotonic. 
It follows from the strict maximum principle (Theorem 2) that each point in the image of $\gamma$ is in $\mathcal{M}^{\prime}$ and that the mean curvature is 0 there. Since $Y \in C \backslash\left(\operatorname{sing} \mathcal{M}^{\prime}\right)$ was arbitrary, this means that

$$
C \times(-\infty, t] \subset \operatorname{spt} \mathcal{M}^{\prime}
$$

Now let $\left(\mathcal{M}^{\prime \prime}, \mathcal{K}^{\prime \prime}\right)$ be a tangent flow at infinity to $\left(\mathcal{M}^{\prime}, \mathcal{K}^{\prime}\right)$ (in other words, a subsequential limit as $r \rightarrow 0$ of $\left.\mathcal{D}_{r}\left(\mathcal{M}^{\prime}, \mathcal{K}^{\prime}\right)\right)$. Then $\left(\mathcal{M}^{\prime \prime}, \mathcal{K}^{\prime \prime}\right)$ is a backward self-similar flow $\mathcal{F}$ and

$$
C^{\prime \prime} \times(-\infty, 0] \subset \operatorname{spt} \mathcal{M}^{\prime \prime}
$$

where $C^{\prime \prime}$ is a tangent cone at infinity to the minimal variety $C$.

By Corollary 2 to Theorem $5, \mathcal{M}^{\prime \prime}$ is completely smooth until time 0 . Thus one connected component must be $C^{\prime \prime} \times(-\infty, 0)$. Since $C^{\prime \prime}$ is a smooth cone, it must be a hyperplane.

Now suppose the portion of the flow $\mathcal{M}^{\prime \prime}$ with $t<0$ had another connected component

$$
\mathcal{N}: t \in(-\infty, 0) \mapsto N_{t} .
$$

Then $N_{t}$ would lie (strictly) to one side of $C^{\prime \prime}$ for $t<0$, but (by self-similarity) would come arbitrarily close to the origin in $C$ as $t \rightarrow 0$. But this would violate the strong half-space theorem below.

Hence $M_{t}^{\prime \prime}$ is $C^{\prime \prime}$ (with multiplicity 1) for $t<0$. It follows [W4 $\left.\$ 5.4\right]$ that $\left(\mathcal{M}^{\prime \prime}, \mathcal{K}^{\prime \prime}\right)$ is a static multiplicity 1 plane. But that implies (by monotonicity) that $\left(\mathcal{M}^{\prime}, \mathcal{K}^{\prime}\right)$ is also a static multiplicity 1 plane.

We end this section by proving the strong half-space theorem, which was used above.

Theorem 7 (strong half-space theorem). Let $\mathcal{M}: t \in[a, b] \mapsto M_{t}$ be a brakke flow (or more generally a weak set flow) of hypersurfaces in $\mathbf{R}^{n+1}$. Suppose there is a closed half-space $H$ such that $H$ contains $M_{a}$ but $M_{a}$ does not contain all of $\partial H$. Then for every $t>a, M_{t}$ is contained in the interior of $H$.

Proof. We may assume that $a=0$ and $H=\left\{x: x_{n+1} \geq 0\right\}$. Shrinking spheres used as barriers show that $M_{t}$ is contained in $H$ for all $t[\mathrm{~B}, \S 3.8]$. Let $f: \mathbf{R}^{n} \rightarrow \mathbf{R}$ be a smooth compactly supported function that is everywhere $\geq 0$ but not everywhere $=0$ and whose graph lies below $M_{a}$. Fix a large ball $\mathbf{B}$ in $\mathbf{R}^{n}$ containing the support of $f$.

Now let

$$
u: \mathbf{B} \times[0, \infty) \rightarrow \mathbf{R}
$$

be the solution of the nonparametric mean curvature flow equation with initial values $u(\cdot, 0)=\phi$ and with boundary values $u(x, t) \equiv 0$ for $x \in \partial \mathbf{B}$. By the strict maximum principle, $u>0$ on the interior of $\mathbf{B} \times[0, \infty)$.

For every $\epsilon>0$, it follows that the graph of

$$
x \mapsto u(x, t)-\epsilon
$$

is disjoint from $M_{t}$ for all $t$. Since this is true for every $\epsilon>0$, we see that (for every $t$ ) the portion of $M_{t}$ in $\mathbf{B} \times \mathbf{R}$ lies in the closed region above the graph of $u(\cdot, t)$.

In particular, (for $t>0$ ) no point of $M_{t}$ in the interior of $\mathbf{B} \times \mathbf{R}$ can touch $\partial H$. Since $\mathbf{B}$ can be arbitrarily large, this establishes the theorem. 


\section{CONVEXITY THEOREMS}

Theorem 8. Let $\left(\mathcal{M}^{\prime}, \mathcal{K}^{\prime}\right)$ be a flow in $\mathcal{F}$ and $X=(x, t)$ be a regular point with $t \leq 0$ and with nonzero mean curvature. Then the principal curvatures of $M_{t}^{\prime}$ at $x$ are all $\geq 0$.

Proof. Let $\gamma$ be the infimum of $G(X)$ over all such regular points $X$ of all such flows $\left(\mathcal{M}^{\prime}, \mathcal{K}^{\prime}\right)$. Let $\left(\mathcal{M}_{i}, \mathcal{K}_{i}\right)$ be a sequence of flows in $\mathcal{F}$ and $X_{i}$ a corresponding sequence of such regular points such that

$$
G\left(\mathcal{M}_{i}, X_{i}\right) \rightarrow \gamma
$$

By translating, we may assume $X_{i} \equiv 0$. By dilating, we may assume

$$
\sup _{|Y|<1} B\left(\mathcal{M}_{i}, Y\right) \leq 1 \leq \sup _{|Y| \leq 1} B\left(\mathcal{M}_{i}, Y\right) .
$$

(The notation is as follows: If $Y=(y, t)$ is a regular point of $\mathcal{M}$, then $B(\mathcal{M}, Y)$ is the norm of the second fundamental form of $M_{t}$ at $y$. If $Y$ is a singular point, $B(\mathcal{M}, Y)$ is infinite.)

By passing to a subsequence, we may assume that the $\left(\mathcal{M}_{i}, \mathcal{K}_{i}\right)$ converge to a limit flow $\left(\mathcal{M}^{\prime}, \mathcal{K}^{\prime}\right)$. Note that the convergence will be smooth on compact subsets of $\{Y:|Y|<1\}$.

Note that $\left(\mathcal{M}^{\prime}, \mathcal{K}^{\prime}\right)$ cannot be a static multiplicity 1 plane. For if it were, then the convergence $\left(\mathcal{M}_{i}, \mathcal{K}_{i}\right) \rightarrow\left(\mathcal{M}^{\prime}, \mathcal{K}^{\prime}\right)$ would be uniform and smooth on compact subsets of spacetime, contradicting $\left(^{*}\right)$.

By Theorem 6, it follows that the mean curvature is strictly positive at every regular point of $\left(\mathcal{M}^{\prime}, \mathcal{K}^{\prime}\right)$ (up to and including time 0 ). In particular, the mean curvature must be strictly positive in $\mathbf{B}^{n+1,1}(0,1)$. Now

$$
G\left(\mathcal{M}^{\prime}, 0\right)=\lim G\left(\mathcal{M}_{i}, 0\right)=\gamma \leq G\left(\mathcal{M}^{\prime}, Y\right)
$$

for every regular $Y=(y, t)$ with $t \leq 0$. Thus the strict maximum principle for $G$ (Theorem 3) implies that $\gamma \geq 0$.

Theorem 9. For each $t \leq 0$, the interior of $K_{t}^{\prime}$ is convex. (If $n<7$, then this holds for all t.)

Proof. First note that the result is true for static flows, since the only static flow in $\mathcal{F}$ is a multiplicity 1 plane (by Theorem 6 ).

Now let $x$ and $y$ be in the interior of $K_{T}^{\prime}$. Then there is an $R>0$ such that

$$
\mathbf{B}(x, R) \cup \mathbf{B}(y, R) \subset \text { interior }\left(K_{T}^{\prime}\right) .
$$

Let $\left(\mathcal{K}^{\prime \prime}, \mathcal{M}^{\prime \prime}\right)$ be the limit flow obtained by applying the translation

$$
(x, t) \in \mathbf{R}^{n+1} \times \mathbf{R} \mapsto(x, t+j)
$$

to $\left(\mathcal{M}^{\prime}, \mathcal{K}^{\prime}\right)$ and then letting $j \rightarrow \infty$. Of course $\left(\mathcal{M}^{\prime \prime}, \mathcal{K}^{\prime \prime}\right)$ is a static flow with

$$
K_{t}^{\prime \prime} \equiv \bigcup_{\tau} K_{\tau}^{\prime} \quad(\forall t)
$$

Since $K_{t}^{\prime \prime}$ is independent of $t$, we omit the subscript. Now since $x$ and $y$ are in the interior of $K^{\prime \prime}$, so is every point in the segment joining $x$ to $y$ (since we know the theorem holds for static flows). Thus for some $\tau<T$,

$$
\overline{x y} \subset \text { interior } K_{\tau}^{\prime} \text {. }
$$


Without loss of generality we may assume that

$$
\begin{aligned}
& x=(-1,0) \in \mathbf{R} \times \mathbf{R}^{n} \cong \mathbf{R}^{n+1}, \\
& y=(1,0) \in \mathbf{R} \times \mathbf{R}^{n} \cong \mathbf{R}^{n+1} .
\end{aligned}
$$

Now let

$$
V=\left\{z \in \mathbf{R}^{n+1}:-1 \leq z_{1} \leq 1,\left|z_{n}\right| \leq \epsilon\left|z_{1}\right|^{2}\right\}
$$

where $\epsilon \subset(0, R)$ is small enough that $V$ is contained in the interior of $K_{\tau}^{\prime}$.

Now we claim that $V \subset K_{T}^{\prime}$. For if not, there would be a first time $t<T$ at which $M_{t}^{\prime}$ touches $V$. By $\left({ }^{*}\right)$, the contact would occur at a point $z$ with $\left|z_{1}\right|<1$.

Note that $(z, t)$ must be a regular point of $\left(\mathcal{M}^{\prime}, \mathcal{K}^{\prime}\right)$. (The proof that it is regular is as follows. Let $\left(\mathcal{M}^{*}, \mathcal{K}^{*}\right)$ be a tangent flow to $\left(\mathcal{M}^{\prime}, \mathcal{K}^{\prime}\right)$ at $(z, t)$. Then

$$
H \subset K_{\tau}^{*}
$$

for $\tau \leq 0$, where $H$ is a closed half-space bounded by $\operatorname{Tan}_{z} M_{t}^{\prime}$. Note that $0 \in$ spt $M_{0}^{*}$. But now, just as in the last two paragraphs of the proof of Theorem 6 , the strong half-space theorem implies $\left(\mathcal{M}^{*}, \mathcal{K}^{*}\right)$ is a static multiplicity 1 plane. Hence $(z, t)$ is a regular point of $\left(\mathcal{M}^{\prime}, \mathcal{K}^{\prime}\right)$.)

But since $V \subset K_{t}^{\prime}$, one of the principal curvatures of $M_{t}^{\prime}$ at $z$ would have to be negative, contradicting Theorem 8.

Corollary. Let $\left(\mathcal{M}^{\prime}, \mathcal{K}^{\prime}\right)$ be a flow in $\mathcal{F}$. Then $K_{t}^{\prime}$ is convex for all $t$.

Proof. By Theorem 5, the regular points form a dense subset of spt $\mathcal{M}^{\prime}$. Each regular point is in the closure of the interior of $\mathcal{K}^{\prime}$. Thus spt $\mathcal{M}^{\prime}$ is contained in the closure of the interior of $\mathcal{K}^{\prime}$. But $\operatorname{spt} \mathcal{M}^{\prime}=\partial \mathcal{K}^{\prime}$ W4 $\left.\S 5.3\right]$. Thus $\partial \mathcal{K}^{\prime}$ is contained in the closure of the interior of $\mathcal{K}^{\prime}$.

Theorem 9 then implies that $K_{t}^{\prime}$ is convex for every $t \leq 0$. But now [W6] implies that $K_{t}^{\prime}$ is convex for all $t$.

Theorem 10. Let $\left(\mathcal{M}^{\prime}, \mathcal{K}^{\prime}\right)$ be a backwardly self-similar flow of convex sets with multiplicity 1 almost everywhere. Then $\left(\mathcal{M}^{\prime}, \mathcal{K}^{\prime}\right)$ is either a static multiplicity 1 plane or a shrinking cylinder. That is, either

$$
K_{t}^{\prime} \equiv K_{-1}^{\prime}=\text { a half-space } H
$$

or else $K_{-1}^{\prime}$ is after a rotation of the form

$$
\mathbf{R}^{k} \times \mathbf{B}^{n+1-k}(0, r)
$$

for some $r>0$ and $k \in\{0,1, \ldots, n-1\}$.

Proof. First, if the flow is static or quasistatic, then $K_{-1}^{\prime}$ is a convex set whose boundary is a minimal cone. This implies that the cone is in fact a plane.

Thus suppose $\left(\mathcal{M}^{\prime}, \mathcal{K}^{\prime}\right)$ is not static or quasistatic. Then every point $(x, t)$ with $t<0$ is a regular point. (Otherwise the tangent flow at $(x, t)$ would be a static counterexample to the theorem.)

Thus $S=M_{-1}^{\prime}$ is a smooth surface that satisfies the equation for self-similarly shrinking surfaces, namely

$$
h(x)=x \cdot \nu
$$

where $h(x)$ is the mean curvature at $x$. (Remark: this equation is equivalent to minimality of $S$ with respect to the riemannian metric $g_{i j}(x)=e^{-|x|^{2} / 2} \delta_{i j}$.) 
This equation together with convexity implies

$$
|A(x)| \leq h(x) \leq|x|
$$

(where $|A(x)|^{2}$ is the sum of the squares of the principal curvatures at $x$ ). Thus if we represent the surface near $x$ as a graph over its tangent plane, the second derivatives are bounded by a constant times $|x|$. The equation (1) becomes a second-order quasielliptic equation, and standard regularity gives estimates on higher derivatives. In particular

$$
|\nabla A(x)| \leq C|x|^{2} .
$$

Now the proof in [H2, §5] shows that $S$ must be a cylinder, provided we check one step: in that proof, there is an integration by parts that must be justified. That is, we must show that the boundary term can be neglected. (In [H2], the second fundamental form of $S$ is known to be bounded, whereas we do not know that here.)

The boundary term is

$$
\int_{\partial(S \cap \mathbf{B}(0, R))}|A|^{2} \nabla\left(\frac{|A|^{2}}{h^{2}}\right) \cdot N \rho
$$

where $N$ is the exterior unit normal to the domain of integration and

$$
\rho(x)=\exp \left(-|x|^{2} / 2\right) .
$$

We need to show that this tends to 0 as $r \rightarrow \infty$. Now

$$
|A|^{2} \nabla\left(\frac{|A|^{2}}{h^{2}}\right)=\left(\frac{|A|^{2}}{h^{2}} \nabla\left(|A|^{2}\right)-\frac{|A|^{4}}{H^{4}} \nabla\left(h^{2}\right)\right) \cdot N .
$$

By (2), this is bounded in norm by

$$
\left|\nabla\left(|A|^{2}\right)\right|+\left|\nabla\left(h^{2}\right)\right| \leq C|x|^{4} .
$$

Thus the integral (3) is bounded by

$$
C r^{4} e^{-r^{2} / 2} \text { area }(\partial(S \cap \mathbf{B}(0, r))
$$

which is bounded by $C r^{3+n} e^{-r^{2} / 2}$ by convexity of $S$. Since this tends to 0 as $r \rightarrow \infty$, we are done.

Theorem 11. Let $\left(\mathcal{M}^{\prime}, \mathcal{K}^{\prime}\right)$ be a flow in $\mathcal{F}$. If $K_{t}^{\prime}$ has nonempty interior, then every point $(x, t)$ in the support of $\mathcal{M}^{\prime}$ is a regular point.

Proof. By hypothesis, there is a ball $\mathbf{B}$ in $K_{t}^{\prime}$. By convexity, the convex hull of $\mathbf{B}$ and $x$ is contained in $K_{t}^{\prime}$. This implies that

$$
\liminf _{r \rightarrow 0} \frac{\mathcal{L}^{n+1}\left(K_{t}^{\prime} \cap \mathbf{B}(x, r)\right)}{\mathcal{L}^{n+1} \mathbf{B}(x, r)}>0 .
$$

Now if $(x, t)$ were a singular point of $\left(\mathcal{M}^{\prime}, \mathcal{K}^{\prime}\right)$, then by the theorem its tangent flows would be shrinking spheres or cylinders, which would force the limit in (4) to be 0 . 


\section{CONCLUSION}

Let $\left(\mathcal{M}^{\prime}, \mathcal{K}^{\prime}\right)$ be a flow in the class $\mathcal{F}$. By Theorem 9 and its corollary, $K_{t}^{\prime}$ is convex for every $t$. By Corollary 1 to Theorem 5 , there is a $T \leq \infty$ such that $K_{t}^{\prime}$ has nonempty interior for $t<T$, and such that $K_{t}^{\prime}$ is empty for $t>T$. By Theorem 11, there are no singular points of $\left(\mathcal{M}^{\prime}, \mathcal{K}^{\prime}\right)$ until time $T$. By Theorem 10, if the flow is backwardly self-similar, then it is a shrinking sphere or cylinder (or a static multiplicity one plane). This completes the proof of Theorem 1 .

To prove the corollary to Theorem 1 , we translate $(\mathcal{M}, \mathcal{K})$ in spacetime by $\left(-x_{i},-t_{i}\right)$ and then dilate parabolically to get $\left(\mathcal{M}_{i}, \mathcal{K}_{i}\right)$ such that

$$
\sup _{|Y|<1} B\left(\mathcal{M}_{i}, Y\right) \leq 1 \leq \sup _{|Y| \leq 1} B\left(\mathcal{M}_{i}, Y\right)
$$

just as was done in the proof of Theorem 8. As in that proof, a subsequence of $\left(\mathcal{M}_{i}, \mathcal{K}_{i}\right)$ (which we may assume to be the original sequence) converges to a limit flow $\left(\mathcal{M}^{\prime}, \mathcal{K}^{\prime}\right)$ in $\mathcal{F}$ in which the origin (of spacetime) is a regular point with strictly positive mean curvature $h$.

Since the origin is a regular point, Theorem 1 implies that $M_{t}^{\prime}$ is smooth and convex until some time $T>0$ (possibly infinite). Brakke's regularity theorem then implies that the convergence

$$
\left(M_{i}\right)_{0} \rightarrow M_{0}^{\prime}
$$

is smooth on compact sets. In particular, the mean curvature of $\left(M_{i}\right)_{0}$ at 0 converges to the mean curvature of $M_{0}^{\prime}$ at 0 .

It follows immediately that that the surfaces $\tilde{M}(i)$ (in the statement of the corollary) converge smoothly on compact sets to $\tilde{M}$, where $\tilde{M}$ is obtained from $M_{0}^{\prime}$ by a dilating by $h$. (This normalizes the mean curvature of $\tilde{M}$ at the origin to be 1.) This completes the proof of the corollary.

\section{Conjectures}

Let $\mathcal{F}$ be as in Theorem 1. Let us call a Brakke flow $\mathcal{M}^{\prime}$ eternal if $M_{t}^{\prime} \neq 0$ for all $t$. Let us call a flow trivial if it is a static multiplicity 1 plane.

Conjecture 1. Any nontrivial eternal flow $\mathcal{M}^{\prime}$ of smooth convex surfaces is a translating solution. That is, there is a vector $v$ such that $M_{t+s}^{\prime}$ is obtained from $M_{t}^{\prime}$ by translating it by $s v$.

Conjecture 2. If $\mathcal{M}^{\prime}$ is an eternal convex translating flow, then (after a rotation) $M_{0}^{\prime}$ has the form

$$
\left\{(x, y, z) \in \mathbf{R}^{j} \times \mathbf{R}^{n-j} \times \mathbf{R}: z=f(|x|)\right\}
$$

where $j \geq 1$.

It is not hard to prove that for each $j \geq 1$, there is an $f$ (unique up to scaling) such that the surface $\left(^{*}\right)$ moves by translation. (If $j=1, f$ is the "grim reaper" curve $f(r)=\ln (\cos r)$ for $-1<r<1$. For $j>1$, the domain of $f$ is all of $\mathbf{R}^{j}$.) The conjecture is that these are the only translating flows.

(As proved in Corollary 4 to Theorem 5 , the $j=1$ example does not actually arise as a limit flow to any mean-convex flow.)

Remark. Even if Conjecture 1 and/or Conjecture 2 are false, they may be true for eternal limit flows to a mean-convex flow. 
Conjecture 3. Suppose $\left(\mathcal{M}^{\prime}, \mathcal{K}^{\prime}\right) \in \mathcal{F}$ is not eternal. Then (after a spacetime translation) it is backwards self-similar (and therefore a shrinking sphere or cylinder).

The assertion is false if we simply assume that $\mathcal{M}^{\prime}$ is a flow of convex surfaces with $M_{t}^{\prime} \neq 0$ for $t<0$ and $M_{t}^{\prime}=0$ for $t>0$. A counterexample is as follows. If $K$ is a compact convex region that is symmetric with respect to $x \mapsto-x$, say that the eccentricity $e(K)$ of $K$ is the ratio of the circumradius to the inradius:

$$
e(K)=\frac{\max \{|x|: x \in K\}}{\min \{|x|: x \in \partial K\}} .
$$

For $j=2,3, \ldots$, consider the mean curvature $(\mathcal{M}(j), \mathcal{K}(j))$ flow generated by such a convex set of eccentricity $j$. By [H1], the eccentricity tends to 1 in finite time. Thus by translating in spacetime and scaling, we may assume that the initial time $t(j)<0$ and that at time $t=0$, the convex set has inradius 1 and circumradius 2 . It is then not hard to show that $t(j) \rightarrow-\infty$ as $j \rightarrow \infty$. Let $\left(\mathcal{M}^{\prime}, \mathcal{K}^{\prime}\right)$ be a subsequential limit of the flows. Then $e\left(K_{0}^{\prime}\right)=2$, so $K_{0}^{\prime}$ is neither a sphere nor a cylinder.

\section{Appendix: Proof of the Strict Maximum Principle for $G$}

Throughout this section, $g=g_{i j}(x, t) d x^{i} \otimes d x^{j}$ will be a time-dependent riemannian metric on $\Omega \times(a, b]$, where $\Omega$ is a connected open subset of $\mathbf{R}^{n}$. Let $\mathbf{v}=v^{i} \frac{\partial}{\partial x^{i}}$ be a vectorfield defined on $\Omega \times(a, b]$. We will say that $\mathbf{v}$ is time-parallel provided

$$
\frac{\partial}{\partial t} v^{i}=-\frac{1}{2} g^{i j}\left(\frac{\partial}{\partial t} g_{j k}\right) v^{k} .
$$

Note that any vectorfield $\mathbf{v}(\cdot, t)$ defined at one time $t$ uniquely determines a timeparallel vectorfield by solving the ODE system (11).

(The equations (11) may seem arbitrary, but in fact they are quite natural. Indeed, they state that $\delta_{\frac{\partial}{\partial t}} \mathbf{v}=0$, where $\delta$ is the Levi-Civita connnection for the riemannian metric $d t \otimes d t+g_{i j} d x^{i} \otimes d x^{j}$ on $\Omega \times\left(a, t_{0}\right]$. We will not, however, explicitly use this connection in what follows.)

Lemma A1. The length of a time-parallel vectorfield $\mathbf{v}(x, \cdot)$ is constant in time.

Note. Here and throughout this appendix, all metric concepts (Laplacian, unit vector, parallel, geodesic) are with respect to the metric $g$.

Proof. Using (11), one readily computes that $\frac{\partial}{\partial t}\left(g_{i j} v^{i} v^{j}\right)=0$. (This also follows immediately from the parenthetical remark above about the Levi-Civita connection.)

The following two propositions are slight variants of [Ha, §8.2].

Proposition A2. Suppose $M=M_{i j}(x, t) d x^{i} \otimes d x^{j}$ is a smooth time-dependent symmetric 2-tensorfield such that

$$
\frac{\partial}{\partial t}(M(\mathbf{v}, \mathbf{v})) \geq(\Delta M)(\mathbf{v}, \mathbf{v})
$$

for all time-parallel vectorfields $\mathbf{v}$. Let $\lambda(x, t)$ be the smallest eigenvalue of $M(x, t)$, i.e., the smallest value of $M(x, t)(\mathbf{u}, \mathbf{u})$ among all unit vectors $\mathbf{u}$. If the minimum value of $\lambda$ on $\Omega \times(a, b]$ occurs at $(x, b)$, then $\lambda$ is constant on $\Omega \times(a, b]$. Furthermore, if $\mathbf{v}$ is an eigenvector of $M(x, t)$ with eigenvalue $\lambda$, then $(\Delta M)(\mathbf{v}, \mathbf{v})=0$. 
Proof. Given a spacetime point $p=(x, t)$, let $\mathbf{v}=\mathbf{v}_{p}$ be a unit vector such that $M(\mathbf{v}, \mathbf{v})=\lambda$. Extend $\mathbf{v}$ to a unit vectorfield $\mathbf{v}(\cdot, t)$ at time $t$ by parallel translation along geodesics emanating from $x$. This way of extending $\mathbf{v}$ guarantees that

$$
(\Delta M)(\mathbf{v}, \mathbf{v})=\Delta(M(\mathbf{v}, \mathbf{v})) \text { at }(x, t) .
$$

Now extend $\mathbf{v}$ as a time-parallel vectorfield on $\Omega \times(a, b]$. Then $\mathbf{v}$ is a unit vectorfield (Lemma A1), so

$$
\lambda \leq M(\mathbf{v}, \mathbf{v}) \text {, with equality at }(x, t) .
$$

Suppose for the moment that $\lambda$ is a smooth function on $\Omega \times(a, b]$. Then by (3) and (21),

$$
\frac{\partial}{\partial t} \lambda=\frac{\partial}{\partial t}(M(\mathbf{v}, \mathbf{v})) \geq(\Delta M)(\mathbf{v}, \mathbf{v})=\Delta(M(\mathbf{v}, \mathbf{v})) \geq \Delta \lambda
$$

at the point $(x, t)$. (The first equality and last inequality are by (3).) Thus if $\lambda$ is smooth, then

$$
\frac{\partial}{\partial t} \lambda \geq \Delta \lambda
$$

Even if $\lambda$ is not smooth, the derivation just given shows that (5) holds in a viscosity sense.

(Remark: in the nonsmooth case, one should think of $\frac{\partial}{\partial t} \lambda$ as

$$
\liminf _{h \rightarrow 0, h>0} \frac{\lambda(x, t)-\lambda(x, t-h)}{h} .
$$

Then by (3), we will still have $\frac{\partial}{\partial t} \lambda \geq \frac{\partial}{\partial t}(M(\mathbf{v}, \mathbf{v}))$ at $(x, t)$.)

The strict maximum principle (cf. Theorem 3, Chapter 5 of $[\mathrm{PW}]$ ) then implies that $\lambda$ is constant.

Now consider the point $(x, t)$ and the special vectorfield $\mathbf{v}$ defined above. Since $\lambda$ is constant, the first and last terms in (4) vanish. This forces all the terms, in particular $(\Delta M)(\mathbf{v}, \mathbf{v})$, to vanish at $(x, t)$.

(Readers unfamiliar with viscosity solutions may wonder how the proof of the strict maximum principle for smooth functions applies to a nonsmooth function such as $\lambda$. The maximum principle for smooth $\lambda$ is proved using smooth functions $u$ such that $u_{t}<\Delta u$ and then observing that it is impossible for $\lambda-u$ to attain a minimum (on certain domains). In the nonsmooth case, note that if $\lambda-u$ attained a minimum at a spacetime point $p$, then for $\mathbf{v}=\mathbf{v}_{p}$, the function $f=M(\mathbf{v}, \mathbf{v})-u$ would also have a minimum at the spacetime point $p$, which readily gives a contradiction since $f$ is a smooth function with $f_{t}>\Delta f$.)

Proposition A3. Assume the hypotheses of Proposition A1, so that $\lambda$ is constant. Then at each time $t, \Omega$ is locally isometric to a product

$$
P \times Q
$$

of two riemannian manifolds $P$ and $Q$, where a vector $\mathbf{v}$ is "horizontal" (i.e., its projection to $Q$ vanishes) if and only it is an eigenvector of $M$ with eigenvalue $\lambda$.

Proof. Without loss of generality, we may assume that $\lambda \equiv 0$; otherwise replace $M$ by $M-\lambda g$. Fix a time $t$. It suffices to prove the conclusion on an open dense subset of $\Omega$. Since the nullity (dimension of the nullspace) of $M$ is locally constant on a dense open subset of $\Omega$, we may assume it is constant throughout $\Omega$. 
Now fix some point $(x, t)$. Let $\mathbf{e}_{i}$ be a $g$-orthonormal basis at $(x, t)$, and extend (spatially) by parallel translation along geodesics emanating from $x$; this guarantees that $\Delta T=\nabla_{e_{i}}\left(\nabla_{e_{i}} T\right)$ for any tensorfield $T$. Now $M(\mathbf{v}, \cdot) \equiv 0$, so

$$
\begin{aligned}
0 & =\Delta(M(\mathbf{v}, \mathbf{v})) \\
& =\nabla_{e_{i}}\left(\nabla_{e_{i}}(M(\mathbf{v}, \mathbf{v}))\right) \\
& =\nabla_{e_{i}}\left(\left(\nabla_{e_{i}} M\right)(\mathbf{v}, \mathbf{v})+2 M\left(\nabla_{e_{i}} \mathbf{v}, \mathbf{v}\right)\right) \\
& =\nabla_{e_{i}}\left(\nabla_{e_{i}} M\right)(\mathbf{v}, \mathbf{v}) \quad(\text { since } M(\cdot, \mathbf{v}) \equiv 0) \\
& =(\Delta M)(\mathbf{v}, \mathbf{v})+2\left(\nabla_{e_{i}} M\right)\left(\nabla_{e_{i}} \mathbf{v}, \mathbf{v}\right) \quad \\
& =2\left(\nabla_{e_{i}} M\right)\left(\nabla_{e_{i}} \mathbf{v}, \mathbf{v}\right) \quad \text { (by Proposition A2) } \\
& =2 \nabla_{e_{i}}\left(M\left(\nabla_{e_{i}} \mathbf{v}, \mathbf{v}\right)\right)-2 M\left(\nabla_{e_{i}} \mathbf{v}, \nabla_{e_{i}} \mathbf{v}\right) \\
& =-2 M\left(\nabla_{e_{i}} \mathbf{v}, \nabla_{e_{i}} \mathbf{v}\right) .
\end{aligned}
$$

Since $M$ is positive semidefinite, this means $\nabla_{e_{i}} \mathbf{v}$ is in the nullspace of $M$ at $(x, t)$ for each $i$. Thus for any vector $X$, the vector $\nabla_{X} \mathbf{v}$ is in the nullspace at $(x, t)$. Since $(x, t)$ is arbitrary, in fact this holds everywhere.

In other words,

$$
\begin{aligned}
& \text { If } \mathbf{v} \text { is a null vectorfield and } X \text { is an arbitrary vectorfield, } \\
& \text { then } \nabla_{X} \mathbf{v} \text { is also a null vectorfield. }
\end{aligned}
$$

By the Frobenius theorem, the nullspaces of $M$ form an integrable distribution. Note by (6) that the leaves of the foliation are totally geodesic.

Now suppose $X$ is an arbitrary vectorfield, $\mathbf{v}$ is a nullvectorfield, and that $\mathbf{u}$ is a vectorfield everywhere perpendicular to the nullvectors. Then

$$
0=\nabla_{X}(\mathbf{u} \cdot \mathbf{v})=\left(\nabla_{X} \mathbf{u}\right) \cdot \mathbf{v}+\mathbf{u} \cdot \nabla_{X} \mathbf{v}=\left(\nabla_{X} \mathbf{u}\right) \cdot \mathbf{v} .
$$

Thus (again by Frobenius) the orthogonal complements of the nullspaces of $M$ form an integrable distribution, and the leaves are totally geodesic.

Thus we can find a coordinate system $\left(y^{1}, \ldots, y^{n}\right)$ such that

$$
g=g_{i j} d y^{i} \otimes d y^{j}+g_{\alpha \beta} d y^{\alpha} \otimes d y^{\beta}
$$

where the Latin indices range from 1 to $m$ (the nullity of $M$ ) and the Greek indices range from $m+1$ to $n$. Since $g_{i \alpha} \equiv 0$, the usual formula [GHL, 2.54] for the Christoffel symbol simplifies to

$$
\Gamma_{i j}^{\alpha}=-\frac{1}{2} g^{\alpha \beta} \partial_{\beta} g_{i j}
$$

Since the horizontal leaves are totally geodesic, $\Gamma_{i j}^{\alpha}$ vanishes for all $\alpha$, which implies that $\partial_{\beta} g_{i j}=0$, so $g_{i j}$ does not depend on $y^{\beta}$. Notice this holds for all $i, j$, and $\beta$. Likewise $g_{\alpha \beta}$ does not depend on any of the $y^{k}$ 's. Thus $g$ is a product metric.

Proposition A4. Let $F: \Omega \times(a, b] \rightarrow \mathbf{R}^{n+1}$ be a classical solution of the mean curvature flow equations, and suppose the mean curvature $h$ is everywhere positive. If the function $G=\kappa_{1} / h$ attains a minimum value of $\gamma$ at $(x, b)$, then $G$ is a nonnegative constant in a spacetime neighborhood of $(x, b)$.

Proof. Let $\mathbf{v}=v^{i} \frac{\partial}{\partial x^{i}}$ be a time-parallel vectorfield. The induced riemannian metric $g$ evolves according to

$$
\frac{\partial}{\partial t} g_{i j}=-2 h A_{i j}
$$


(see [H1]), where $A$ is the second fundamental form, so

$$
\frac{\partial}{\partial t} v^{i}=h g^{i j} A_{j k} v^{k}
$$

The second fundamental form evolves according to

$$
\frac{\partial}{\partial t} A_{i j}=\Delta A_{i j}+|A|^{2} A_{i j}-2 h A_{i p} g^{p q} A_{q j}
$$

(by [H1]), so

$$
\begin{aligned}
\frac{\partial}{\partial t} A(\mathbf{v}, \mathbf{v}) & =\frac{\partial}{\partial t}\left(A_{i j} v^{i} v^{j}\right) \\
& =\left(\frac{\partial}{\partial t} A_{i j}\right) v^{i} v^{j}+2 A_{i j}\left(\frac{\partial}{\partial t} v^{i}\right) \mathbf{v}^{j} \\
& =\left(\Delta A_{i j}+|A|^{2} A_{i j}-2 h A_{i p} g^{p q} A_{q j}\right) v^{i} v^{j}-2 A_{i j} h g^{i k} A_{k \ell} v^{\ell} v^{j} \\
& =\left(\Delta A_{i j}+|A|^{2} A_{i j}\right) v^{i} v^{j} .
\end{aligned}
$$

Similarly,

(by [H1]), so

$$
\frac{\partial}{\partial t} h=\Delta h+|A|^{2} h
$$

$$
\begin{array}{rlr}
\frac{\partial}{\partial t} h g(\mathbf{v}, \mathbf{v}) & =\left(\frac{\partial}{\partial t} h\right) g(\mathbf{v}, \mathbf{v}) & \text { (by Lemma A1) } \\
& =\left(\Delta h+|A|^{2} h\right) g(\mathbf{v}, \mathbf{v}) & \\
& =\left(\left(\Delta+|A|^{2}\right)(h g)\right)(\mathbf{v}, \mathbf{v})
\end{array}
$$

where the last line is by parallelism of $g$ (so that $\Delta(h g)=(\Delta h) g)$. Combining (7) and (8) shows that the tensorfield $M=A-\gamma h g$ satisfies

$$
\frac{\partial}{\partial t}(M(\mathbf{v}, \mathbf{v}))=(\Delta M)(\mathbf{v}, \mathbf{v})+|A|^{2} M(\mathbf{v}, \mathbf{v}) \geq(\Delta M)(\mathbf{v}, \mathbf{v}) .
$$

This last inequality holds since $M$ is positive semidefinite (by choice of $\gamma$ ). Note that the first eigenvalue $\lambda=\kappa_{1}-\gamma h$ of $M$ is everywhere nonnegative and is 0 at $(x, b)$. Thus by Proposition A2, $\lambda$ is identically 0 .

Fix a time $t$. Then $\Omega$ is locally a metric product $P \times Q$ (by Proposition A3). Let $X$ and $Y$ be unit eigenvectors of $A$ (at some given point) with eigenvalues $\kappa_{1}$ and $\kappa_{n}$, respectively, and assume that $\kappa_{1} \leq 0$. Then $\kappa_{n}>0$ since $h>0$. Thus $X$ and $Y$ will be horizontal and vertical, respectively, with respect to the product structure $P \times Q$ (by Proposition A3).

The sectional curvature determined by $X$ and $Y$ is given by Gauss's theorem GHL, 5.5]:

$$
A(X, X) A(Y, Y)-A(X, Y)^{2}=\kappa_{1} \kappa_{n} .
$$

On the other hand, this sectional curvature must vanish because the metric is a product metric (see [GHL, 3.15]). Since $\kappa_{n}$ is positive, $\kappa_{1}$ must vanish.

\section{REFERENCES}

[B] K. Brakke, The motion of a surface by its mean curvature, Princeton U. Press, 1978. MR 82c:49035

[GHL] S. Gallot, D. Hulin, and J. Lafontaine, Riemannian geometry, Springer-Verlag, 1987. MR 88k:53001

[Ha] R. S. Hamilton, Four-manifolds with positive curvature operator, J. Differential Geom. 24 (1986), 153-179. MR 87m:53055 
[H1] G. Huisken, Flow by mean curvature of convex surfaces into spheres, J. Differential Geom. 20 (1984), 237-266. MR 86j:53097

[H2] Local and global behavior of hypersurfaces moving by mean curvature, Proc. Symp. Pure Math. 54 (1993), 175-191. MR 94c:58037.

[HS1] G. Huisken and C. Sinestrari, Mean curvature flow singularities for mean convex surfaces, Calc. Var. Partial Differential Equations 8 (1999), 1-14. MR 99m:58057]

[HS2] - Convexity estimates for mean curvature flow and singularities of mean convex surfaces, Acta Math. 183 (1999), 45-70. MR 2001c:53094

[I1] T. Ilmanen, Elliptic regularization and partial regularity for motion by mean curvature, Mem. Amer. Math. Soc. 108 (520) (1994). MR 95d:49060

[I2] The level-set flow on a manifold, Proc. Symp. Pure Math. 54 (1993), 193-204. MR 94d:58040

[I3] Singularities of mean curvature flow of surfaces, preprint.

[PW] M. H. Protter and H. F. Weinberger, Maximum principles in differential equations, Springer-Verlag, 1984. MR 86f:35034

[SJ] J. Simons, Minimal varieties in riemannian manifolds, Ann. of Math. 88 (1962), 62-105. MR 38:1617

[SS] R. Schoen and L. Simon, Regularity of stable minimal hypersurfaces, Comm. Pure Appl. Math. 34 (1981), 741-797. MR 82k:49054

[W1] Brian White, Partial regularity of mean-convex hypersurfaces flowing by mean curvature, International Math. Research Notices 4 (1994), 185-192. MR 95b:58042

[W2] The topology of hypersurfaces moving by mean curvature, Comm. Analysis and Geom. 3 (1995), 317-333. MR 96k:58051

[W3] Stratification of minimal surfaces, mean curvature flows, and harmonic maps, J. reine angew. Math. 488 (1997), 1-35. MR 99b:49038

[W4] , The size of the singular set in mean curvature flow of mean-convex surfaces, J. Amer. Math. Soc. 13 (2000), 665-695. MR 2001j:53098

[W5] Brakke's regularity theorem for limits of smooth flows, in preparation.

[W6] - A strict maximum principle at infinity for mean curvature flow, in preparation.

Department of Mathematics, Stanford University, Stanford, California 94305-2060

E-mail address: white@math.stanford.edu 